\title{
Measurable Spaces and their Effect Logic
}

\author{
Bart Jacobs \\ Institute for Computing and Information Sciences (iCIS), Radboud University Nijmegen, The Netherlands. \\ Web address: www.cs.ru.nl/B.Jacobs Email: bart@cs.ru.nl
}

\begin{abstract}
So-called effect algebras and modules are basic mathematical structures that were first identified in mathematical physics, for the study of quantum logic and quantum probability. They incorporate a double negation law $p^{\perp \perp}=p$. Since then it has been realised that these effect structures form a useful abstraction that covers not only quantum logic, but also Boolean logic and probabilistic logic. Moreover, the duality between effect and convex structures lies at the heart of the duality between predicates and states. These insights are leading to a uniform framework for the semantics of computation and logic. This framework has been elaborated elsewhere for settheoretic, discrete probabilistic, and quantum computation. Here the missing case of continuous probability is shown to fit in the same uniform framework. On a technical level, this involves an investigation of the logical aspects of the Giry monad on measurable spaces and of Lebesgue integration.
\end{abstract}

Keywords-Probabilistic system, measurable space, Giry monad, effect algebra, duality.

\section{INTRODUCTION}

Edsger Dijkstra invented the weakest pre-condition calculus as a systematic technique for deriving program properties, see [1]. For a program/statement $s$ the calculus involves an operation $\operatorname{wp}(s)$ that transform a post-condition $Q$ into the weakest pre-condition $P=\operatorname{wp}(s)(Q)$ that guarantees that $Q$ holds in the "post" state resulting from executing $s$ in a "pre" state where $P$ holds. More mathematically, for nondeterministic program going from state $X$ to state $Y$, the weakest pre-condition calculus involves bijective correspondences between:

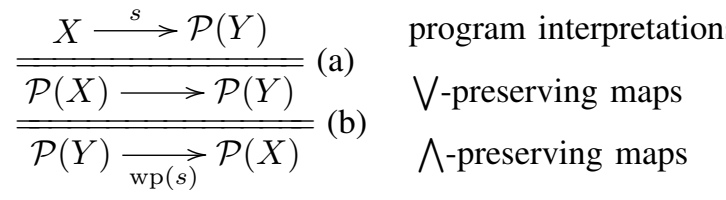

The latter map, involving a reversal of $X$ and $Y$, computes the weakest pre-condition from the post-condition.

More categorically, this can be expressed via a diagram:

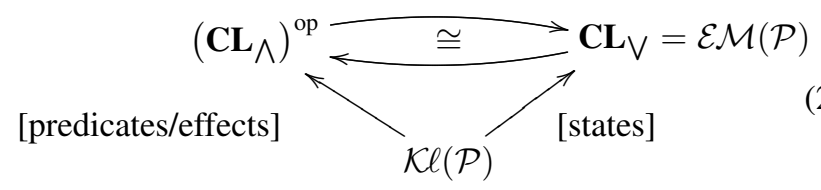

Here $\mathbf{C} \mathbf{L}_{\Lambda}$ is the category of complete lattices with maps preserving all meets $\bigwedge$. Similarly, in $\mathbf{C L} \vee$ maps preserve all joins. This category is the Eilenberg-Moore category of the powerset monad $\mathcal{P}$. At the bottom there is the Kleisli category

\footnotetext{
To appear in the proceedings of LICS 2013.
}

$\mathcal{K} \ell(\mathcal{P})$ with maps of the form $X \rightarrow \mathcal{P}(Y)$ as interpretations of non-deterministic programs. The full \& faithful functor $\mathcal{K} \ell(\mathcal{P}) \rightarrow \mathcal{E} \mathcal{M}(\mathcal{P})$ gives the above correspondence (a) in (1); the correspondence (b) in (1) arises from the basic fact that $\bigvee$ preserving maps between complete lattices have $\bigwedge$-preserving adjoints.

This diagram (2) incorporates a duality that is similar to the duality between states and predicates/effects, which, in a quantum setting is associated with the different approaches of Schrödinger (states) and Heisenberg (effects) [2], see below. In computer science this corresponds to the difference between weakest pre-condition operations (working backwards) and strongest post-condition operations (working forwards).

Indeed, this picture (2) captures the essence of the semantics of program logics, also for probabilistic and quantum computation. In [3] it is shown that for discrete probabilistic computations, represented via the monad $\mathcal{D}$ of discrete probability distributions, there is a diagram:

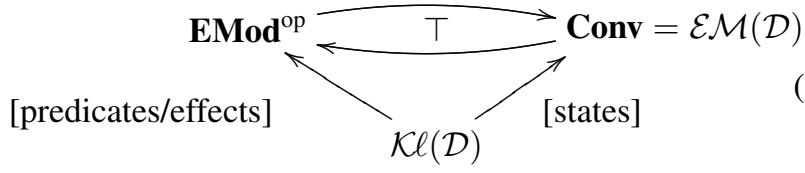

States are now represented by the category Conv of convex sets, and predicates by the category EMod of effect modules (see Subsection II-A). In the quantum case the picture is similar but the base category involves Hilbert spaces (and isometries as maps, see [3] for details):

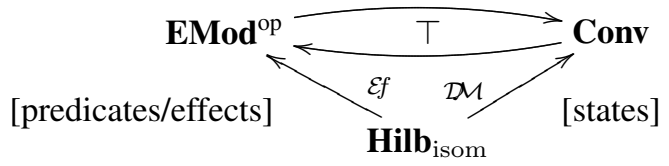

The main result of the current paper shows that this same diagram also occurs for continuous probabilistic computation/logic. It leads to a correspondence as in (1), like in Kozen's duality [4], as will be shown in the very end.

Theorem 1: For the Giry monad $\mathcal{G}$ on the category Meas of measurable spaces there is a diagram:

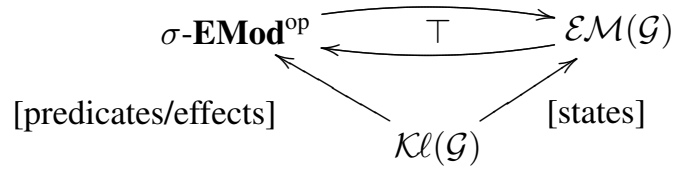

The subcategory $\sigma$-EMod $\hookrightarrow$ EMod contains $\omega$-continuous effect modules, with joins of increasing chains.

The main contributions of this paper are: 
- An extension of the uniform framework for program semantics and logic proposed in [3]; the main examples used there involve set-theoretic, discrete probabilistic, and quantum computation. Here we elaborate the missing case of continuous probabilistic computation.

- Identification of the relevant probabilistic predicates, by proving the correspondence between measurable maps $X \rightarrow[0,1]$ and "decidable" predicates $p: X \rightarrow X+X$ with $\nabla \circ p=\mathrm{id}$ - in $\mathcal{K} \ell(\mathcal{G})$ - as used in [3] for logics with double negation. Additionally, "characteristic" maps for (quantum-style) measurement, for dynamic logical operations "andthen" and "then", and for probability density functions are identified in this setting.

- A re-discovery of Kozen's duality [4] in a more systematic and general setting.

- Promotion of "effect" structures as the relevant logical structures covering Boolean, probabilistic, and also quantum logic. This promotion includes a systematic account of Lebesgue integration and the Giry monad in terms of these effect structures.

In the end one may view the current work as a precise elaboration of Lawvere's early ideas (see e.g. [5]) about the analogies between logic in terms of subsets and union (via the powerset monad) and logic in terms of measurable maps and integration (first elaborated by Giry [6] and many others [4], [7], [8], [9], [10], [11], [12], [13], [14], [15]).

This paper is organised as follows. After describing the mathematical preliminaries it proceeds with the correspondence between measurable and decidable predicates. Sections $\mathrm{IV}$ and $\mathrm{V}$ provide alternative formulations, of the Giry monad in terms of predicates, and of predicates in terms of the Giry monad. This resembles the quantum situation with Gleason-style isomorphisms $\operatorname{Hom}(\mathcal{E} f(H),[0,1]) \cong \mathcal{D} M(H)$ and $\operatorname{Hom}(\mathcal{D M}(H),[0,1]) \cong \mathcal{E} f(H)$, relating effects and density matrices, see [16], [17]. The final section VI connects predicates and states, leading to the main result (Theorem 1 .

\section{MATHEMATICAL PRELIMINARIES}

This section prepares the ground, by introducing in three separate subsections the basics of effect algebras/modules, of Lebesgue integration and of the Giry monad on the category of measurable spaces. We assume familiarity with basic category theory, including the theory of monads.

\section{A. Effect algebras and effect modules}

Effect algebras have been introduced in mathematical physics [18], in the investigation of quantum probability, see [19] for an overview. An effect algebra is a partial commutative monoid $(M, 0, \otimes)$ with an orthocomplement $(-)^{\perp}$. One writes $x \perp y$ if $x \oslash y$ is defined. The orthocomplement must satisfy two requirements: (1) $x^{\perp}$ is unique with $x \oslash x^{\perp}=1$, where $1=0^{\perp}$, and (2) $x \perp 1$ implies $x=0$. Each effect algebra is partially ordered, by $x \leq y$ iff $x \otimes z=y$, for some $z$. The main example is the unit interval $[0,1] \subseteq \mathbb{R}$, where addition + is obviously partial, commutative, associative, and has 0 as unit; moreover, the orthocomplement is $r^{\perp}=1-r$.
A $\sigma$-effect algebra additionally has joins $\bigvee x_{n}$ of countable chains $x_{0} \leq x_{1} \leq \cdots$. In the current setting we assume all effect algebras are such $\sigma$-effect algebras (so we omit the ' $\sigma$ ' in (1)). We write EA for the category of $\sigma$-effect algebras, with morphism preserving $\emptyset, 1, \bigvee$.

For each set $X$, the set $[0,1]^{X}$ of fuzzy predicates on $X$ is an effect algebra, via pointwise operations. Each ( $\omega$-complete) Boolean algebra $B$ is an effect algebra with $x \perp y$ iff $x \wedge y=$ $\perp$; then $x \otimes y=x \vee y$. Interestingly, George Boole originally defined union for disjoint subsets only. In a quantum setting, the main example is the set of effects $\mathcal{E} f(H)=\{E: H \rightarrow$ $H \mid 0 \leq E \leq I\}$ on a Hilbert space $H$, see e.g. [19], [2].

An effect module is an "effect" version of a vector space. It involves an effect algebra $M$ with a scalar multiplication $s \bullet x \in M$, where $s \in[0,1]$ and $x \in M$. This scalar multiplication is required to be a suitable homomorphism in each variable separately. The algebras $[0,1]^{X}$ and $\mathcal{E} f(H)$ are clearly such effect modules. In the subcategory EMod $\hookrightarrow$ EA maps additionally commute with scalar multiplication. Since our effect algebras have joins $\bigvee$, so do effect modules.

We need the (finite, discrete probability) distribution monad $\mathcal{D}:$ Sets $\rightarrow$ Sets. It sends a set $X$ to the set $\mathcal{D}(X)=$ $\left\{\varphi: X \rightarrow[0,1] \mid \operatorname{supp}(\varphi)\right.$ is finite, and $\left.\sum_{x} \varphi(x)=1\right\}$, where $\operatorname{supp}(\varphi)=\{x \mid \varphi(x) \neq 0\}$. Such an element $\varphi \in \mathcal{D}(X)$ may be identified with a formal finite convex sum $\sum_{i} r_{i} x_{i}$ with $x_{i} \in X$ and $r_{i} \in[0,1]$ satisfying $\sum_{i} r_{i}=1$. A convex set is an Eilenberg-Moore algebra of this monad: it consists of a carrier set $X$ in which actual sums $\sum_{i} r_{i} x_{i} \in X$ exist for all convex combinations. Like in Diagram (3) we write Conv $=\mathcal{E} \mathcal{M}(\mathcal{D})$ for the category of convex sets, with "affine" functions preserving convex sums.

Effect modules and convex sets are related via a basic adjunction [17], obtained by "homming into [0,1]", as in:

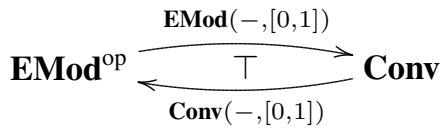

\section{B. Measurable spaces and the Giry functor}

A measurable space - i.e. an object of the category Meas — is a pair $X=\left(X, \Sigma_{X}\right)$ consisting of a set $X$ together with a $\sigma$-algebra $\Sigma_{X} \subseteq \mathcal{P}(X)$. The latter is a collection of "measurable" subsets closed under $\emptyset$, complements (negation), and countable unions. This set $\Sigma_{X}$ forms a Boolean algebra - and hence an effect algebra - in which countable joins exist. A measurable space $X$ is called discrete if $\Sigma_{X}=\mathcal{P}(X)$, where $X$ is either finite or countable.

A morphism $X \rightarrow Y$ in Meas, from $\left(X, \Sigma_{X}\right)$ to $\left(Y, \Sigma_{Y}\right)$, is a measurable function $f: X \rightarrow Y$, i.e. a function satisfying $f^{-1}(M) \in \Sigma_{X}$ for each measurable subset $M \in \Sigma_{Y}$. This yields a functor Meas $\rightarrow \mathbf{E} \mathbf{A}^{\mathrm{op}}$, given by $X \mapsto \Sigma_{X}$. With each topological space $X$ with opens $\mathcal{O}(X)$ one associates the least $\sigma$-algebra containing $\mathcal{O}(X)$. This is the Borel algebra/space on $X$. In particular the unit interval $[0,1]$ forms a measurable space. Its measurable subsets are generated by the intervals $[q, 1]$, where $q$ is a rational number in $[0,1]$. 
Given measurable spaces $Y_{i}$ and functions $f_{i}: X \rightarrow Y_{i}$ there is a least $\sigma$-algebra $\Sigma_{X} \subseteq \mathcal{P}(X)$ making all functions $f_{i}$ measurable. Thus $\Sigma_{X}$ contains all $f_{i}^{-1}(M)$ for $M \in \Sigma_{Y_{i}}$.

The (categorical) product $X_{1} \times X_{2}$ of two measurable spaces $X_{i}$ carries the least $\sigma$-algebra making both projections $\pi_{i}: X_{1} \times X_{2} \rightarrow X_{i}$ measurable functions; equivalently, this $\sigma$-algebra is generated by the rectangles $M_{1} \times M_{2}$ with $M_{i} \in \Sigma_{X_{i}}$ The coproduct $X_{1}+X_{2}$ involves the disjoint union of the underlying sets with the $\sigma$-algebra given by the direct images $\kappa_{i} M=\left\{\kappa_{i} x \mid x \in M\right\}$ for $M \in \Sigma_{X_{i}}$, where $\kappa_{i}: X_{i} \rightarrow X_{1}+X_{2}$ is the coprojection map.

A measure space consists of a measurable space $X=$ $\left(X, \Sigma_{X}\right)$ together with a function $\phi: \Sigma_{X} \rightarrow \mathbb{R}_{\geq 0}$ which satisfies $\phi(\emptyset)=0$ and is countably additive:

$$
\phi\left(\bigotimes_{i \in I} M_{i}\right)=\sum_{i \in I} \phi\left(M_{i}\right),
$$

for each pairwise disjoint, countable collection of measurable subsets $M_{i} \in \Sigma_{X}$. Here we use $\otimes$ for disjoint union, where $\Sigma_{X}$ is understood as effect algebra. Such a function $\phi$ is called a measure. This measure $\phi$ is called a probability measure if $\phi(X)=1$, so that $\phi$ can be restricted to a function $\Sigma_{X} \rightarrow$ $[0,1]$, and forms a map of effect algebras. In that case the triple $\left(X, \Sigma_{X}, \phi\right)$ is called a probability space.

We now describe the Giry functor $\mathcal{G}$ : Meas $\rightarrow$ Meas, introduced in [6]. For a measurable space $X \in$ Meas we set:

$$
\mathcal{G}(X)=\left\{\phi: \Sigma_{X} \rightarrow[0,1] \mid \phi \text { is a probability measure }\right\} .
$$

Each measurable subset $M \in \Sigma_{X}$ yields a function $\mathrm{ev}_{M}: \mathcal{G}(X) \rightarrow[0,1]$, namely ev $v_{M}(\phi)=\phi(M)$. Thus one can equip the set $\mathcal{G}(X)$ with the least $\sigma$-algebra making all these maps $\mathrm{ev}_{M}$ measurable. We obtain a functor Meas $\rightarrow$ Meas since for a map $f: X \rightarrow Y$ in Meas we get a measurable function $\mathcal{G}(f): \mathcal{G}(X) \rightarrow \mathcal{G}(Y)$ given by:

$$
\mathcal{G}(f)\left(\Sigma_{X} \stackrel{\phi}{\rightarrow}[0,1]\right)=\left(\Sigma_{Y} \stackrel{f^{-1}}{\rightarrow} \Sigma_{X} \stackrel{\phi}{\rightarrow}[0,1]\right) .
$$

For a probability measure $\phi$ on $X \times Y$ one gets a probability measure $\mathcal{G}\left(\pi_{1}\right)(\phi)$ on $X$, which is the marginal of $\phi$. It is given on $M \in \Sigma_{X}$ by:

$$
\mathcal{G}\left(\pi_{1}\right)(\phi)(M)=\phi\left(\pi_{1}^{-1}(M)\right)=\phi(M \times Y) .
$$

Probability measures are closed under convex sums, making $\mathcal{G}(X)$ a convex set: for a finite collection $\phi_{i} \in \mathcal{G}(X)$ and $r_{i} \in[0,1]$ with $\sum_{i} r_{i}=1$ one has $\sum_{i} r_{i} \phi_{i} \in \mathcal{G}(X)$.

\section{Lebesgue integration and the Giry monad}

Let $\left(X, \Sigma_{X}, \phi\right)$ be a probability space, as described above, so that $\phi \in \mathcal{G}(X)$. We will use integration only for measurable functions $X \rightarrow[0,1]$, with the unit interval as codomain, and not for more general real- or complex-valued functions. These functions $X \rightarrow[0,1]$ may be understood as $[0,1]$ valued stochastic variables - or as "measurable predicates", as we shall see in Section III Therefor we write $\operatorname{Pred}(X)=$ $\operatorname{Meas}(X,[0,1])$. These sets $\operatorname{Pred}(X)$ are effect modules, with $p \perp q$ if $p(x)+q(x) \leq 1$ for all $x \in X$. In that case one defines $(p \otimes q)(x)=p(x)+q(x)$. The orthocomplement is given by $p^{\perp}(x)=1-p(x)$ and scalar multiplication by $(s \bullet p)(x)=s \cdot p(x)$. The top element is $\lambda x .1$ and the bottom is $\lambda x .0$. Notice that when $X$ is a discrete space, the set of predicates $\operatorname{Pred}(X)$ is the set $[0,1]^{X}$ of all functions $X \rightarrow[0,1]$, which is the set of fuzzy predicates used in the discrete probabilistic case investigated in [3].

For each $M \in \Sigma_{X}$ we write $\mathbf{1}_{M}: X \rightarrow[0,1]$ for the indicator function given by $\mathbf{1}_{M}(x)=1$ for $x \in M$ and $\mathbf{1}_{M}(x)=0$ for $x \notin M$. A step function is a finite linear combination $r_{1} \mathbf{1}_{M_{1}}+\cdots+r_{k} \mathbf{1}_{M_{k}}=\bigotimes_{i} r_{i} \bullet \mathbf{1}_{M_{i}} \in \operatorname{Pred}(X)$ of indicator functions with $r_{i} \in[0,1]$ and $M_{i} \in \Sigma_{X}$ pairwise disjoint measurable subsets. A first observation is that each measurable predicate can be approximated (from below) by step functions.

Lemma 2: For each map $p: X \rightarrow[0,1]$ in Meas there is a sequence of step functions $p_{n} \leq p$ so that $p$ can be written both as:

- pointwise join $p=\bigvee_{n \in \mathbb{N}} p_{n}$;

- limit $p=\lim _{n \rightarrow \infty} p_{n}$ of a uniformly convergent sequence.

Proof Following [17] we define $p_{n}(x)=0 . d_{1} d_{2} \cdots d_{n}$, where $d_{i}$ is the $i$-th decimal of $p(x) \in[0,1]$. This $p_{n}$ takes at most $10^{n}$ different values, since $d_{i} \in\{0,1, \ldots, 9\}$. For each of these values $r_{i} \in[0,1]$ there is a measurable subset $M_{i}=p^{-1}\left(\left\{r_{i}\right\}\right) \in \Sigma_{X}$, since the singleton subset/interval $\left\{r_{i}\right\}=\left[r_{i}, r_{i}\right] \subseteq[0,1]$ is measurable. Thus we can write $p_{n}=\sum_{i} r_{i} \mathbf{1}_{M_{i}}$, so that it is a step function.

By construction, $p_{n} \leq p$. For each $\epsilon>0$, take $N \in \mathbb{N}$ such that for all decimals $d_{i}$ we have:

$$
0 . \underbrace{00 \cdots 00}_{N \text { times }} d_{1} d_{2} d_{3} \cdots<\epsilon .
$$

Then for each $n \geq N$ we have $p(x)-p_{n}(x)<\epsilon$, for all $x \in X$, and thus $d\left(p, p_{n}\right) \leq \epsilon$. Hence $\bigvee_{n} p_{n}=p$ and $p=\lim _{n \rightarrow \infty} p_{n}$.

Next we summarise the main steps in defining the (Lebesgue) integral for measurable predicates.

Definition 3: Let $\left(X, \Sigma_{X}, \phi\right)$ be a probability space.

i) For $M \in \Sigma_{X}$ the integral of the associated indicator function is defined as:

$$
\int \mathbf{1}_{M} \mathrm{~d} \phi=\phi(M) \in[0,1] .
$$

ii) This definition is extended linearly to step functions:

$$
\int\left(\sum_{i} r_{i} \mathbf{1}_{M_{i}}\right) \mathrm{d} \phi=\sum_{i} r_{i} \phi\left(M_{i}\right) \in[0,1] .
$$

(This sum is in [0,1] since: $\sum_{i} r_{i} \phi\left(M_{i}\right) \leq \sum_{i} \phi\left(M_{i}\right)=$ $\phi\left(\otimes_{i} M_{i}\right) \leq \phi(X)=1$.

iii) Next, this integral is extended continuously to all measurable functions $p: X \rightarrow[0,1]$; after writing them as limit $p=\lim p_{n}$ of step functions $p_{n}$ like in Lemma 2 one defines: $\stackrel{n \rightarrow \infty}{\rightarrow}$

$$
\int p \mathrm{~d} \phi=\lim _{n \rightarrow \infty} \int p_{n} \mathrm{~d} \phi \in[0,1] .
$$

This integral $\int p \mathrm{~d} \phi$ is sometimes written as $E[p]$, since it describes the expectation value of the predicate $p$. 
We list some basic properties of integration.

Lemma 4: Let $X$ be an arbitrary measurable space.

i) For each $\phi \in \mathcal{G}(X)$ the operation $p \mapsto \int p \mathrm{~d} \phi$ is a map of effect modules $\operatorname{Pred}(X) \rightarrow[0,1]$ that preserves pointwise limits.

ii) For a map $f: X \rightarrow Y$ in Meas and predicate $q: Y \rightarrow$ $[0,1]$,

$$
\int(q \circ f) \mathrm{d} \phi=\int q \mathrm{~d} \mathcal{G}(f)(\phi) .
$$

iii) For each $x \in X$ and $p \in \operatorname{Pred}(X)$ one has:

$$
\int p \mathrm{~d} \eta(x)=p(x)
$$

where $\eta_{X}: X \rightarrow \mathcal{G}(X)$ is the unit map given by:

$$
\eta_{X}(x)(M)=\mathbf{1}_{M}(x) .
$$

This unit $\eta$ yields a natural transformation $\eta$ : id $\Rightarrow \mathcal{G}$.

The next definition introduces two operations that are of fundamental importance in this setting.

Definition 5: With an arbitrary measurable function $f: X \rightarrow \mathcal{G}(Y)$ we associate two operations:

i) "Kleisli extension" $f^{\$}: \mathcal{G}(X) \rightarrow \mathcal{G}(Y)$, given by:

$$
\begin{aligned}
f^{\Phi}(\phi)(N) & =\int f(-)(N) \mathrm{d} \phi \\
& =\int(\lambda x \in X . f(x)(N)) \mathrm{d} \phi .
\end{aligned}
$$

This uses that for $N \in \Sigma_{Y}$ one has a measurable function $f(-)(N): X \rightarrow[0,1]$.

ii) "Substitution" $f^{*}: \operatorname{Pred}(Y) \rightarrow \operatorname{Pred}(X)$ given by:

$$
f^{*}(q)=\int q \mathrm{~d} f(-)=\lambda x \in X . \int q \mathrm{~d} f(x) .
$$

Since integration $\int(-) \mathrm{d} \phi$ is a limit-preserving map of effect modules (see Lemma 4), so is the substitution map $f^{*}$, in a pointwise manner.

These operations of Kleisli extension $f^{\mathscr{S}}$ and substitution $f^{*}$ are related in a basic manner, resembling a Galois connection. This seemingly new observation gives a short proof of Theorem 7.

Proposition 6: For each map $f: X \rightarrow \mathcal{G}(Y)$ in Meas, probability measure $\phi \in \mathcal{G}(Y)$ and predicate $q \in \operatorname{Pred}(Y)$ one has:

$$
\int f^{*}(q) \mathrm{d} \phi=\int q \mathrm{~d} f^{\$}(\phi)
$$

Proof Because of limit-preservation of substitution and integration it suffices to prove the result for predicates given by step functions $s=\sum_{i} r_{i} \mathbf{1}_{N_{i}} \in \operatorname{Pred}(Y)$. Then:

$$
\begin{aligned}
\int f^{*}(s) \mathrm{d} \phi & =\int f^{*}\left(\sum_{i} r_{i} \mathbf{1}_{N_{i}}\right) \mathrm{d} \phi \\
& =\int \sum_{i} r_{i} f^{*}\left(\mathbf{1}_{N_{i}}\right) \mathrm{d} \phi \\
& \text { since } f^{*} \text { is a map of effect modules } \\
& \stackrel{\text { 10 }}{=} \sum_{i} r_{i} \int\left(\lambda x \cdot \int \mathbf{1}_{N_{i}} \mathrm{~d} f(x)\right) \mathrm{d} \phi \\
& =\sum_{i} r_{i} \int f(-)\left(N_{i}\right) \mathrm{d} \phi \\
& \stackrel{9}{=} \sum_{i} r_{i} f^{\$}(\phi)\left(N_{i}\right) \\
& =\sum_{i} r_{i} \int \mathbf{1}_{N_{i}} \mathrm{~d} f^{\$}(\phi) \\
& =\int \sum_{i} r_{i} \mathbf{1}_{N_{i}} \mathrm{~d} f^{\Phi}(\phi) \\
& =\int s \mathrm{~d} f^{\$}(\phi) .
\end{aligned}
$$

We are finally in a position to see that $\mathcal{G}$ is a monad. We do so by following the formulation in terms of Kleisli extension.

Theorem 7 (From [6]): The functor $\mathcal{G}:$ Meas $\rightarrow$ Meas is a monad, with unit $\eta$ from $(8)$ and Kleisli extension $(-)^{\$}$ from 9 .

Proof We check the equations for Kleisli extension: the unit equations $\eta^{\$}=\mathrm{id}$ and $f^{\$} \circ \eta=f$ are obtained as follows.

$$
\begin{aligned}
& \eta^{\$}(\phi)(M)=\int \eta(-)(M) \mathrm{d} \phi \\
& \text { (8) } \int \mathbf{1}_{M} \mathrm{~d} \phi \\
& =\phi(M) \\
& \left(f^{\Phi} \circ \eta\right)(x)(N)=f^{\Phi}(\eta(x))(N) \\
& \text { 包 } \int f(-)(N) \mathrm{d} \eta(x) \\
& \text { 그 } f(x)(N) \text {. }
\end{aligned}
$$

The composition equation $g^{\$} \circ f^{\$}=\left(g^{\$} \circ f\right)^{\$}$ requires a bit more care:

$$
\begin{aligned}
\left(g^{\$} \circ f^{\$}\right)(\phi)(K) & =g^{\$}\left(f^{\$}(\phi)\right)(K) \\
& \underline{\underline{\theta}} \int g(-)(K) \mathrm{d} f^{\$}(\phi) \\
& =\int f^{*}(g(-)(K)) \mathrm{d} \phi \\
& \quad \text { by Proposition } 6 \\
& \stackrel{\underline{10}}{=} \int\left(\lambda x \cdot \int g(-)(K) \mathrm{d} f(x)\right) \mathrm{d} \phi \\
& \underline{\underline{9}} \int\left(\lambda x \cdot g^{\$}(f(x))(K)\right) \mathrm{d} \phi \\
& =\int\left(g^{\$} \circ f\right)(-)(K) \mathrm{d} \phi \\
& =\left(g^{\$} \circ f\right)^{\$}(\phi)(K) .
\end{aligned}
$$

As a result, composition in the Kleisli category $\mathcal{K} \ell(\mathcal{G})$ is given as follows. For $f: X \rightarrow \mathcal{G}(Y)$ and $g: Y \rightarrow \mathcal{G}(Z)$ we have:

$$
(g \circ f)(x)(K)=\int g(-)(K) \mathrm{d} f(x)
$$

where $x \in X$ and $K \in \Sigma_{Z}$.

The multiplication $\mu: \mathcal{G}^{2}(X) \rightarrow \mathcal{G}(X)$ of the monad is given on $\Phi \in \mathcal{G}^{2}(X)$ and $M \in \Sigma_{X}$ by:

$$
\begin{aligned}
\mu(\Phi)(M)=\left(\operatorname{id}_{\mathcal{G}(X)}\right)^{\Phi}(\Phi)(M) & =\int \operatorname{id}(-)(M) \mathrm{d} \Phi \\
& =\int \mathrm{ev}_{M} \mathrm{~d} \Phi .
\end{aligned}
$$


The following observation is sometimes useful.

Lemma 8: For $p \in \operatorname{Pred}(X)$ and $\Phi \in \mathcal{G}^{2}(X)$ one has

$$
\int p \mathrm{~d} \mu(\Phi)=\int\left(\lambda \phi . \int p \mathrm{~d} \phi\right) \mathrm{d} \Phi .
$$

Proof By Proposition 6

$$
\begin{aligned}
\int p \mathrm{~d} \mu(\Phi) \stackrel{12}{=} \int p \mathrm{did}^{\Phi}(\Phi) & =\int \mathrm{id}^{*}(p) \mathrm{d} \Phi \\
& \stackrel{10}{=} \int\left(\lambda \phi . \int p \mathrm{~d} \phi\right) \mathrm{d} \Phi .
\end{aligned}
$$

The Giry monad is commutative, via a map dst: $\mathcal{G}(X) \times$ $\mathcal{G}(Y) \rightarrow \mathcal{G}(X \times Y)$; for probability measures $\phi: \Sigma_{X} \rightarrow$ $[0,1]$ and $\phi: \Sigma_{Y} \rightarrow[0,1]$ we get a probability measure $\operatorname{dst}(\phi, \psi): \Sigma_{X \times Y} \rightarrow[0,1]$ determined by $\operatorname{dst}(\phi, \psi)(M \times N)=$ $\phi(M) \times \psi(N)$. In particular, the strength map st: $\mathcal{G}(X) \times Y \rightarrow$ $\mathcal{G}(X \times Y)$ is given by $\operatorname{st}(\phi, y)(M \times N)=\phi(M) \cdot \mathbf{1}_{N}(y)$. As a result, the product $\times$ of measurable spaces becomes a tensor $\otimes$ on the Kleisli category $\mathcal{K} \ell(\mathcal{G})$. The tensor unit is the singleton (discrete) measurable space $1=\{0\}$, with $\Sigma_{1}=\{\emptyset, 1\}$.

On this tensor unit we have:

$\mathcal{G}(1)=\left\{\phi: \Sigma_{1} \rightarrow[0,1] \mid \phi\right.$ is a probability measure $\} \cong 1$,

since $\phi(\emptyset)=0$ and $\phi(1)=1$. Hence there is precisely one element in $\mathcal{G}(1)$. This makes $\mathcal{G}$ an affine monad.

\section{PRedicates}

In "quantitative" logics as used in probability and quantum theory double negation is essential. For this purpose predicates with this double negation built-in are represented in [3] as maps $f: X \rightarrow X+X$ in $\nabla \circ f=$ id, where $\nabla$ is the codiagonal given by the cotuple $\nabla=[\mathrm{id}, \mathrm{id}]: X+X \rightarrow X$. This definition makes sense in a category with coproducts + and leads to effect module structure on the collection of predicates on $X$, provided the coproducts satisfy some elementary properties. We call such predicates 'decidable', because that is how they are called in a topos. Below we interpret these predicate in the Kleisli category $\mathcal{K} \ell(\mathcal{G})$ of the Giry monad and show that such decidable predicates on $X$ corresponds to measurable maps $X \rightarrow[0,1]$, i.e. to $[0,1]$ valued random/stochastic variables. Earlier we have already used the notation $\operatorname{Pred}(X)$ for the set of these maps. We have seen that predicates carry the structure of an effect module, and that this structure is preserved by substitution.

A predicate following [3] in $\mathcal{K} \ell(\mathcal{G})$ is thus a map $f: X \rightarrow$ $\mathcal{G}(X+X)$ in Meas with $\mathcal{G}(\nabla) \circ f=\eta$. Hence, for $x \in X$ and $M \in \Sigma_{X}$ we have $f(x)\left(\nabla^{-1}(M)\right)=\eta(x)(M)$. Since

$$
\begin{aligned}
\nabla^{-1}(M) & =\{z \in X+X \mid \nabla(z) \in M\} \\
& =\left\{\kappa_{1} x \mid x \in M\right\} \otimes\left\{\kappa_{2} x \mid x \in M\right\} \\
& =\kappa_{1} M \otimes \kappa_{2} M
\end{aligned}
$$

and $f(x)$ is a probability measure, the map $f$ satisfies:

$$
f(x)\left(\kappa_{1} M\right)+f(x)\left(\kappa_{2} M\right)= \begin{cases}1 & \text { if } x \in M \\ 0 & \text { otherwise. }\end{cases}
$$

Thus such $f$ is determined by elements $x \in M$ for $M \in \Sigma_{X}$.

An elementary but crucial observation about decidable predicates is the following "splitting" result.

Lemma 9: For a map $f: X \rightarrow X+X$ satisfying $\nabla \circ f=$ id in $\mathcal{K} \ell(\mathcal{G})$ one has, for each $x \in X$ and $M \in \Sigma_{X}$,

$$
\begin{aligned}
& f(x)\left(\kappa_{1} M\right)=f(x)\left(\kappa_{1} X\right) \cdot \mathbf{1}_{M}(x) \\
& f(x)\left(\kappa_{2} M\right)=f(x)\left(\kappa_{2} X\right) \cdot \mathbf{1}_{M}(x) .
\end{aligned}
$$

Proof We shall do the " $\kappa_{1}$ " case. When $x \notin M$ the equation $f(x)\left(\kappa_{1} M\right)=f(x)\left(\kappa_{1} X\right) \cdot \mathbf{1}_{M}(x)$ holds because both sides are 0 , by (13). And when $x \in M$, then $x \notin \neg M$, so $f(x)\left(\kappa_{1} \neg M\right)=0$, again by (13). Hence:

$$
\begin{aligned}
f(x)\left(\kappa_{1} X\right) & =f(x)\left(\kappa_{1} M \oslash \kappa_{1} \neg M\right) \\
& =f(x)\left(\kappa_{1} M\right)+f(x)\left(\kappa_{1} \neg M\right) \\
& =f(x)\left(\kappa_{1} M\right) .
\end{aligned}
$$

Proposition 10: There is a bijective correspondence between decidable predicates on $X \in \mathcal{K} \ell(\mathcal{G})$ and measurable predicates $X \rightarrow[0,1]$.

Proof Starting from $f: X \rightarrow \mathcal{G}(X+X)$ satisfying (13) we define $p_{f}: X \rightarrow[0,1]$ by:

$$
p_{f}(x)=f(x)\left(\kappa_{1} X\right) .
$$

This $p_{f}$ is measurable, since for $r \in[0,1]$,

$$
\begin{aligned}
\left(p_{f}\right)^{-1}([r, 1]) & =\left\{x \in X \mid p_{f}(x) \in[r, 1]\right\} \\
& =\left\{x \in X \mid f(x)\left(\kappa_{1} X\right) \in[r, 1]\right\} \\
& =\left\{x \in X \mid \mathrm{ev}_{\kappa_{1} X}(f(x)) \in[r, 1]\right\} \\
& =\left\{x \in X \mid f(x) \in \mathrm{ev}_{\kappa_{1} X}^{-1}([r, 1])\right\} \\
& =f^{-1}\left(\mathrm{ev}_{\kappa_{1} X}^{-1}([r, 1])\right) .
\end{aligned}
$$

The latter is in $\Sigma_{X}$ because $f$ is a measurable function.

In the other direction, starting from a measurable function $p: X \rightarrow[0,1]$ we define $f_{p}: X \rightarrow \mathcal{G}(X+X)$ via:

$$
\begin{aligned}
& f_{p}(x)\left(\kappa_{1} M\right)=p(x) \cdot \mathbf{1}_{M}(x)= \begin{cases}p(x) & \text { if } x \in M \\
0 & \text { otherwise. }\end{cases} \\
& f_{p}(x)\left(\kappa_{2} M\right)=(1-p(x)) \cdot \mathbf{1}_{M}(x)= \begin{cases}1-p(x) & \text { if } x \in M \\
0 & \text { otherwise. }\end{cases}
\end{aligned}
$$

By construction, the equation (13) holds. We have to check that $f_{p}$ is measurable. For $\kappa_{1} M \in \Sigma_{X+X}$ and $r \in[0,1]$ we get:

$$
\begin{aligned}
f_{p}^{-1}\left(\mathrm{ev}_{\kappa_{1} M}^{-1}([r, 1])\right) & =\left\{x \in X \mid f_{p}(x) \in \mathrm{ev}_{\kappa_{1} M}^{-1}([r, 1])\right\} \\
& =\left\{x \in X \mid f_{p}(x)\left(\kappa_{1} M\right) \in[r, 1]\right\} \\
& = \begin{cases}X & \text { if } r=0 \\
M \cap\{x \mid p(x) \in[r, 1]\} & \text { if } r>0\end{cases} \\
& = \begin{cases}X & \text { if } r=0 \\
M \cap p^{-1}([r, 1]) & \text { if } r>0 .\end{cases}
\end{aligned}
$$

In both cases this yields a measurable subset of $X$. The " $\kappa_{2}$ "case works similarly. 
Finally, we prove that the two constructions $f \mapsto p_{f}$ and $p \mapsto f_{p}$ are each other's inverses.

$$
\begin{array}{rlr}
f_{p_{f}}(x)\left(\kappa_{1} M\right) & =p_{f}(x) \cdot \mathbf{1}_{M}(x) \\
& =f(x)\left(\kappa_{1} X\right) \cdot \mathbf{1}_{M}(x) & \\
& =f(x)\left(\kappa_{1} M\right) & \\
f_{p_{f}}(x)\left(\kappa_{2} M\right) & =\left(1-p_{f}(x)\right) \cdot \mathbf{1}_{M}(x) & \\
& =\left(1-f(x)\left(\kappa_{1} X\right)\right) \cdot \mathbf{1}_{M}(x) \\
& =f(x)\left(\kappa_{2} X\right) \cdot \mathbf{1}_{M}(x) \quad \text { bemma } 9 \\
& =f(x)\left(\kappa_{2} M\right) & \\
p_{f_{p}}(x) & =f_{p}(x)\left(\kappa_{1} X\right) \\
& =p(x) \cdot \mathbf{1}_{X}(x) \\
& =p(x) .
\end{array}
$$

As before we write $\operatorname{Pred}(X)=\operatorname{Meas}(X,[0,1]) \cong$ $\mathcal{K} \ell(\mathcal{G})(X, 2)$ and consider the elements $p \in \operatorname{Pred}(X)$ as (measurable) predicates on $X$. We freely use the previous lemma to switch between the two equivalent formulations of predicates. The mapping $X \mapsto \operatorname{Pred}(X)$ yields a functor Pred: $\mathcal{K} \ell(\mathcal{G}) \rightarrow$ EMod $^{\mathrm{op}}$, given on morphisms by substitution $f^{*}$ as in (10). Such a functor is also called an indexed category and forms a basic structure in categorical logic [20].

We recall from [3] that on objects of the form $X+X$ a special decidable predicate exists, namely: $\Omega_{X}=\kappa_{1}+$ $\kappa_{2}: X+X \rightarrow(X+X)+(X+X)$. Alternatively, it can be described as measurable map $\Omega_{X}=\mathbf{1}_{\kappa_{1} X}: X+X \rightarrow[0,1]$.

Lemma 11: For each predicate $p \in \operatorname{Pred}(X)$ there is a map $\operatorname{char}_{p}: X \rightarrow X+X$ in $\mathcal{K} \ell(\mathcal{G})$ with $\left(\operatorname{char}_{p}\right)^{*}\left(\Omega_{X}\right)=p$.

Proof Take $\operatorname{char}_{p}=f_{p}$ as defined in the proof of Proposition 10 . Then for $x \in X$,

$$
\begin{aligned}
\left(\operatorname{char}_{p}\right)^{*}\left(\Omega_{X}\right)(x) & =\int \Omega_{X} \mathrm{~d} \operatorname{char}_{p}(x)=\int \mathbf{1}_{\kappa_{1} X} \mathrm{~d} f_{p}(x) \\
& =f_{p}(x)\left(\kappa_{1} X\right) \\
& \stackrel{133}{=} p(x) \cdot \mathbf{1}_{X}(x)=p(x) .
\end{aligned}
$$

In [3] it is shown that these characteristic maps $\operatorname{char}_{p}$ play a crucial role in (quantum-like) measurement. They are also essential to define the basic operations of a dynamic logic with measurable predicates. But first we need an auxiliary result about integration over a coproduct space $X+Y$.

Lemma 12: For two measurable spaces $X, Y$ with a probability measure $\phi \in \mathcal{G}(X+Y)$ and predicate $p \in \operatorname{Pred}(X+Y)$ one can split the integral over $X+Y$ into a convex sum of two integrals, over $X$ and $Y$ separately:

$$
\begin{aligned}
\int p \mathrm{~d} \phi=\phi\left(\kappa_{1} X\right) & \cdot \int\left(p \circ \kappa_{1}\right) \mathrm{d} \frac{\phi\left(\kappa_{1}(-)\right)}{\phi\left(\kappa_{1} X\right)} \\
& +\phi\left(\kappa_{2} Y\right) \cdot \int\left(p \circ \kappa_{2}\right) \mathrm{d} \frac{\phi\left(\kappa_{2}(-)\right)}{\phi\left(\kappa_{2} Y\right)},
\end{aligned}
$$

where on the right-hand-side of the equality sign $=$ the first summand is 0 if $\phi\left(\kappa_{1} X\right)=0$ and similarly the second summand is 0 if $\phi\left(\kappa_{2} Y\right)=0$.
Proof We first note that the probability measure $\phi: \Sigma_{X+Y} \rightarrow$ $[0,1]$ satisfies:

$$
\phi\left(\kappa_{1} X\right)+\phi\left(\kappa_{2} Y\right)=\phi\left(\kappa_{1} X \otimes \kappa_{2} Y\right)=\phi(X+Y)=1 .
$$

Hence the sum in the lemma is indeed a convex one. This measure $\phi$ can be split into two probability measures $\phi_{1} \in$ $\mathcal{G}(X)$ and $\phi_{2} \in \mathcal{G}(Y)$, namely:

$$
\begin{array}{ll}
\Sigma_{X} \stackrel{\phi_{1}=\frac{\phi\left(\kappa_{1}(-)\right)}{\phi\left(\kappa_{1} X\right)}}{\longrightarrow}[0,1] & \Sigma_{Y} \stackrel{\phi_{2}=\frac{\phi\left(\kappa_{2}(-)\right)}{\phi\left(\kappa_{2} Y\right)}}{\longrightarrow}[0,1] \\
M \longmapsto & \stackrel{\phi\left(\kappa_{1} M\right)}{\phi\left(\kappa_{1} X\right)} \quad N \longmapsto \frac{\phi\left(\kappa_{2} N\right)}{\phi\left(\kappa_{2} Y\right)},
\end{array}
$$

using the direct images $\kappa_{1} M=\left\{\kappa_{1} x \mid x \in M\right\}$ and $\kappa_{2} N=$ $\left\{\kappa_{1} y \mid y \in N\right\}$. Of course, this only works when $\phi\left(\kappa_{1} X\right) \neq 0$ or $\phi\left(\kappa_{1} Y\right) \neq 0$, but if one of them is 0 , the other one is 1 .

We prove the lemma for step functions on $X+Y$ and observe that such a step function $s=\left(\sum_{i \in I} r_{i} \mathbf{1}_{K_{i}}\right) \in$ $\operatorname{Pred}(X+Y)$ can be written as cotuple $s=\left[s_{1}, s_{2}\right]$ where:

- $s_{1}=\left(\sum_{i \in I_{1}} r_{i} \mathbf{1}_{M_{i}}\right) \in \operatorname{Pred}(X)$ with $\kappa_{1} M_{i}=K_{i}$ for $i \in I_{1}$

- $s_{2}=\left(\sum_{i \in I_{2}} r_{i} \mathbf{1}_{N_{i}}\right) \in \operatorname{Pred}(Y)$ with $\kappa_{2} N_{i}=K_{i}$ for $i \in I_{2}$

- $I=I_{1} \otimes I_{2}$.

Then:

$$
\begin{aligned}
\phi & \left(\kappa_{1} X\right) \cdot \int\left(s \circ \kappa_{1}\right) \mathrm{d} \phi_{1}+\phi\left(\kappa_{2} Y\right) \cdot \int\left(s \circ \kappa_{2}\right) \mathrm{d} \phi_{2} \\
& =\phi\left(\kappa_{1} X\right) \cdot \int s_{1} \mathrm{~d} \phi_{1}+\phi\left(\kappa_{2} Y\right) \cdot \int s_{2} \mathrm{~d} \phi_{2} \\
& =\phi\left(\kappa_{1} X\right) \cdot \sum_{i \in I_{1}} r_{i} \phi_{1}\left(M_{i}\right)+\phi\left(\kappa_{2} Y\right) \cdot \sum_{i \in I_{2}} r_{i} \phi_{2}\left(N_{i}\right) \\
& =\sum_{i \in I_{1}} r_{i} \phi\left(\kappa_{1} M_{i}\right)+\sum_{i \in I_{2}} r_{i} \phi\left(\kappa_{2} N_{i}\right) \\
& =\sum_{i \in I_{1}} r_{i} \phi\left(K_{i}\right)+\sum_{i \in I_{2}} r_{i} \phi\left(K_{i}\right) \\
& =\sum_{i \in I} r_{i} \phi\left(K_{i}\right) \\
& =\int s \mathrm{~d} \phi .
\end{aligned}
$$

Now that we have the identified the effect module structure on predicates, substitution, and characteristic maps, we can interpret the dynamic logic operations "andthen" $\langle p ?\rangle(q)$ and "then" $[p ?](q)$ from [3]. In abstract terms they are defined for decidable predicates $p, q: X \rightarrow X+X$ as:

$$
\begin{aligned}
\langle p ?\rangle(q) & =\left(\operatorname{char}_{p}\right)^{*}\left[\left(\kappa_{1}+\kappa_{1}\right) \circ q, \kappa_{2} \circ \kappa_{2}\right] \\
{[p ?](q) } & =\left(\operatorname{char}_{p}\right)^{*}\left[\left(\kappa_{1}+\kappa_{1}\right) \circ q, \kappa_{1} \circ \kappa_{2}\right] .
\end{aligned}
$$

Proposition 13: For measurable predicates $p, q \in \operatorname{Pred}(X)=\operatorname{Meas}(X,[0,1])$ the definitions (14) translate into:

$$
\begin{aligned}
\langle p ?\rangle(q) & =\lambda x \cdot p(x) \cdot q(x) \\
{[p ?](q)(x) } & =\lambda x \cdot p(x) \cdot q(x)+1-p(x)=\langle p ?\rangle(q) \otimes p^{\perp} .
\end{aligned}
$$

These formulas correspond to the ones for fuzzy predicates $X \rightarrow[0,1]$ in Sets, in the context of discrete probability 
theory described in [3]. The first formula correspond to the sequential composition operation on effect algebras from [21], which in the case of effects $E, D$ on Hilbert spaces is given by $\langle E$ ? $\rangle(D)=\sqrt{E} D \sqrt{E}$, see also [3]. The last formula for $[p ?](q)(x)$ gives the so-called Reichenbach implication [22].

Proof We shall do the calculations for $[p ?](q)(x)$, for $p, q: X \rightarrow[0,1]$. The decidable predicate $\left[\left(\kappa_{1}+\kappa_{1}\right) \circ f_{q}, \kappa_{2} \circ\right.$ $\left.\kappa_{1}\right]: X+X \rightarrow \mathcal{G}((X+X)+(X+X))$ corresponds to the measurable predicate $r \in[0,1]^{X+X}$ given by:

$$
r\left(\kappa_{1} x\right)=q(x) \quad r\left(\kappa_{2} x\right)=1 .
$$

Then, using Lemmas 12 and 9 , we get:

$$
\begin{aligned}
& {[p ?](q)(x)=\left(f_{p}\right)^{*}\left[\left(\kappa_{1}+\kappa_{1}\right) \circ f_{q}, \kappa_{1} \circ \kappa_{2}\right](x)} \\
& \text { [10] } \int r \mathrm{~d} f_{p}(x) \\
& =f_{p}(x)\left(\kappa_{1} X\right) \cdot \int\left(r \circ \kappa_{1}\right) \mathrm{d} \frac{f_{p}(x)\left(\kappa_{1}(-)\right)}{f_{p}(x)\left(\kappa_{1} X\right)} \\
& +f_{p}(x)\left(\kappa_{2} X\right) \cdot \int\left(r \circ \kappa_{2}\right) \mathrm{d} \frac{f_{p}(x)\left(\kappa_{2}(-)\right)}{f_{p}(x)\left(\kappa_{2} X\right)} \\
& =p(x) \cdot \int q \mathrm{~d} \frac{f_{p}(x)\left(\kappa_{1} X\right) \cdot \mathbf{1}_{-}(x)}{f_{p}(x)\left(\kappa_{1} X\right)} \\
& +(1-p(x)) \cdot \int \mathbf{1}_{X} \mathrm{~d} \frac{f_{p}(x)\left(\kappa_{2} X\right) \cdot \mathbf{1}_{-}(y)}{f_{p}(x)\left(\kappa_{2} X\right)} \\
& =p(x) \cdot \int q \mathrm{~d} \eta(x)+(1-p(x)) \cdot \int \mathbf{1}_{X} \mathrm{~d} \eta(x) \\
& =p(x) \cdot q(x)+(1-p(x)) .
\end{aligned}
$$

The characteristic maps $\operatorname{char}_{p}: X \rightarrow X+X$ in $\mathcal{K}(\mathcal{G})$ are also useful for describing probability density functions (pdf's): the Kleisli extension char $_{p}^{\$}: \mathcal{G}(X) \rightarrow \mathcal{G}(X+X)$ is given by:

$$
\begin{aligned}
\operatorname{char}_{p}^{\$}(\phi)\left(\kappa_{1} M\right) & =\int \operatorname{char}_{p}(-)\left(\kappa_{1} M\right) \mathrm{d} \phi \\
\operatorname{char}_{p}^{\$}(\phi)\left(\kappa_{2} M\right) & =\iint_{M} p \mathrm{~d} \phi \\
\operatorname{char}_{p}(-)\left(\kappa_{2} M\right) \mathrm{d} \phi & =\int_{M} p^{\perp} \mathrm{d} \phi .
\end{aligned}
$$

Thus $p$ is pdf for the measure $M \mapsto \operatorname{char}_{p}^{\$}(\phi)\left(\kappa_{1} M\right)$.

\section{THE GIRY MONAD IN TERMS OF PREDICATES}

In this section we prove that the Giry monad can be expressed in terms of predicates. In the next section it will be shown that the converse also holds.

Lemma 14: For each $X \in$ Meas we define a function:

$$
\begin{aligned}
\operatorname{EMod}(\operatorname{Pred}(X),[0,1]) \longrightarrow \theta_{X} & >\mathcal{G}(X) \\
h \longmapsto & \longrightarrow \Sigma_{X} \cdot h\left(\mathbf{1}_{M}\right) .
\end{aligned}
$$

Then:

i) For each predicate $p \in \operatorname{Pred}(X)$,

$$
\int p \mathrm{~d} \theta_{X}(h)=h(p) .
$$

ii) Each $\theta_{X}$ is an isomorphism, with inverse:

$$
\left(\theta_{X}\right)^{-1}(\phi)(p)=\int p \mathrm{~d} \phi
$$

iii) These $\theta_{X}$ are natural in $X \in \mathcal{K} \ell(\mathcal{G})$ - and their inverses too.

Proof We first have to check that $\theta(h)$ is well-defined, i.e. forms a probability measure. Since $h$ is a map of effect algebras, it preserves bottom and top, and thus:

$$
\begin{aligned}
\theta(h)(\emptyset) & =h\left(\mathbf{1}_{\emptyset}\right)=h(0)=0 \\
\theta(h)(X) & =h\left(\mathbf{1}_{X}\right)=h(1)=1 .
\end{aligned}
$$

For a collection $\left(M_{i}\right)_{i \in \mathbb{N}}$ of pairwise disjoint measurable subsets of $X$, put $N_{i}=M_{0} \otimes \cdots \otimes M_{i} \in \Sigma_{X}$. These $N_{i}$ then form an ascending chain, and so do the indicator functions $\mathbf{1}_{N_{i}} \in \operatorname{Pred}(X)$. Using that $h$ preserves joins, we get:

$$
\begin{aligned}
\theta(h)\left(\bigotimes_{i} M_{i}\right)=h\left(\mathbf{1}_{\bigotimes_{i} M_{i}}\right) & =h\left(\emptyset_{i} \mathbf{1}_{M_{i}}\right) \\
& =h\left(\bigvee_{i} \mathbf{1}_{N_{i}}\right) \\
& =\bigvee_{i} h\left(\mathbf{1}_{N_{i}}\right) \\
& =\bigvee_{i} h\left(\mathbf{1}_{M_{0}} \oslash \cdots \otimes \mathbf{1}_{M_{i}}\right) \\
& =\bigvee_{i} h\left(\mathbf{1}_{M_{0}}\right)+\cdots+h\left(\mathbf{1}_{M_{i}}\right) \\
& =\sum_{i} h\left(\mathbf{1}_{M_{i}}\right) .
\end{aligned}
$$

We now turn to the three points in the lemma.

i) We first prove the equation for a step function $s=$ $\sum_{i} r_{i} \mathbf{1}_{M_{i}} \in \operatorname{Pred}(X)$. Since $h$ is a map of effect modules we get:

$$
\begin{aligned}
\int s \mathrm{~d} \theta(h) & =\sum_{i} r_{i} \theta(h)\left(M_{i}\right)=\sum_{i} r_{i} h\left(\mathbf{1}_{M_{i}}\right) \\
& =h\left(\emptyset_{i} r_{i} \mathbf{1}_{M_{i}}\right)=h(s) .
\end{aligned}
$$

For an arbitrary predicate $p \in \operatorname{Pred}(X)$, approximated by stepfunctions $s_{n}$, we have, because $h$ preserves joins:

$$
\begin{aligned}
\int p \mathrm{~d} \theta(h) & =\bigvee_{n} \int s_{n} \mathrm{~d} \theta(h) \\
& =\bigvee_{n} h\left(s_{n}\right)=h\left(\bigvee_{n} s_{n}\right)=h(p) .
\end{aligned}
$$

ii) We first note that $\theta^{-1}$ is well-defined since $\theta^{-1}(\phi)=$ $\int(-) \mathrm{d} \phi: \operatorname{Pred}(X) \rightarrow[0,1]$ is a map of effect modules by Lemma 4 (i). The equation $\theta^{-1} \circ \theta=$ id amounts to $\int p \mathrm{~d} \theta(h)=h(p)$, which is point (i). For the reverse equation $\theta \circ \theta^{-1}=$ id we simply calculate:

$$
\theta\left(\theta^{-1}(\phi)\right)(M)=\theta^{-1}(\phi)\left(\mathbf{1}_{M}\right)=\int \mathbf{1}_{M} \mathrm{~d} \phi=\phi(M) .
$$

iii) For naturality, let $f: X \rightarrow \mathcal{G}(Y)$. Then for $h: \operatorname{Pred}(X) \rightarrow[0,1]$ and $N \in \Sigma_{Y}$ one has:

$$
\begin{aligned}
\left(f^{\$} \circ \theta_{X}\right)(h)(N) & =f^{\Phi}\left(\theta_{X}(h)\right)(N) \\
& \underline{\underline{ }} \int f(-)(N) \mathrm{d} \theta_{X}(h) \\
& \stackrel{(i)}{=} h(f(-)(N)) \\
& =h(\lambda x \cdot f(x)(N)) \\
& =h\left(\lambda x \cdot \int \mathbf{1}_{N} \mathrm{~d} f(x)\right) \\
& \stackrel{10}{=} h\left(f^{*}\left(\mathbf{1}_{N}\right)\right) \\
& =\theta_{Y}\left(h \circ f^{*}\right)(N) \\
& =\left(\theta_{Y} \circ\left((-) \circ f^{*}\right)\right)(h)(N) .
\end{aligned}
$$


The expectation monad on Sets, given by $X \mapsto$ $\operatorname{EMod}\left([0,1]^{X},[0,1]\right)$ is investigated in [23]. The analogous mapping $X \mapsto \operatorname{EMod}(\operatorname{Pred}(X),[0,1])$, for $X \in \operatorname{Meas,~may~}$ be seen as a measurable/continuous version of this expectation monad. The previous lemma shows that this is the Giry monad $\mathcal{G}$ on the category of measurable functions.

In the discrete case there is an analogue of Lemma 14 for finite sets $X$; it says $\operatorname{EMod}\left([0,1]^{X},[0,1]\right) \cong \mathcal{D}(X)$, see [23]. The quantum analogue relates effects and density matrices on a finite-dimensional Hilbert space $H$, via $\operatorname{EMod}(\mathcal{E} f(H),[0,1]) \cong \mathcal{D M}(H)$, see [16], [17].

\section{PRedicates In terms of The GiRy MONAD}

We start with some investigations in the category $\mathcal{E} \mathcal{M}(\mathcal{G})$ of Eilenberg-Moore algebras of the Giry monad.

Lemma 15: The unit interval $[0,1] \in$ Meas carries an Eilenberg-Moore algebra structure $\alpha: \mathcal{G}([0,1]) \rightarrow[0,1]$ given by $\alpha(\phi)=\int \mathrm{id} \mathrm{d} \phi$.

A measurable function $g: \mathcal{G}(X) \rightarrow[0,1]$ is an algebra homomorphism if and only if it satisfies $g(\phi)=\int(g \circ \eta) \mathrm{d} \phi$.

Proof We check the two algebra equations $\alpha \circ \eta=$ id and $\alpha \circ \mu=\alpha \circ \mathcal{G}(\alpha)$. Clearly, $(\alpha \circ \eta)(x)=\int \mathrm{id} \mathrm{d} \eta(x)=$ $\operatorname{id}(x)=x$, by (7). Further, for $\Phi \in \mathcal{G}^{2}(X)$,

$$
\begin{array}{rlr}
(\alpha \circ \mu)(\Phi) & =\int \operatorname{idd} \mu(\Phi) & \\
& =\int\left(\lambda \phi \cdot \int \operatorname{idd} \phi\right) \mathrm{d} \Phi & \text { by Lemma } 8 \\
& =\int \alpha \mathrm{d} \Phi \\
& =\int \operatorname{idd} \mathcal{G}(\alpha)(\Phi) \quad \text { by } 6 \\
& =\alpha(\mathcal{G}(\alpha)(\Phi)) & \\
& =(\alpha \circ \mathcal{G}(\alpha))(\Phi) .
\end{array}
$$

Let $g: \mathcal{G}(X) \rightarrow[0,1]$ be a map in Meas. It is an algebra map if and only if $g \circ \mu_{X}=\alpha \circ \mathcal{G}(g)$. This means, for $\Phi \in \mathcal{G}^{2}(X)$,

$$
g(\mu(\Phi))=\alpha(\mathcal{G}(g))(\Phi)=\int \operatorname{idd} \mathcal{G}(g)(\Phi) \underline{\underline{6}} \int g \mathrm{~d} \Phi .
$$

By using the monad equation $\mu \circ \mathcal{G}(\eta)=$ id we now get:

$$
g(\phi)=g(\mu(\mathcal{G}(\eta)(\phi)))=\int g \mathrm{~d} \mathcal{G}(\eta)(\phi) \stackrel{6}{\underline{6}} \int(g \circ \eta) \mathrm{d} \phi .
$$

Conversely, assuming this equation, the map $g$ is an algebra homomorphism:

$$
\begin{aligned}
g(\mu(\Phi)) & =\int(g \circ \eta) \mathrm{d} \mu(\Phi) & & \text { by assumption } \\
& =\int\left(\lambda \phi \cdot \int(g \circ \eta) \mathrm{d} \phi\right) \mathrm{d} \Phi & & \text { by Lemma } 8 \\
& =\int g \mathrm{~d} \Phi & & \text { by assumption. }
\end{aligned}
$$

Lemma 16: For each $X \in$ Meas there is a map:

$$
\begin{aligned}
\mathcal{E M}(\mathcal{G})(\mathcal{G}(X),[0,1]) \stackrel{\vartheta_{X}}{\longrightarrow} & \operatorname{Pred}(X) \\
g \longmapsto & g \circ \eta .
\end{aligned}
$$

It is a natural isomorphism, with inverse $\vartheta_{X}^{-1}(p)(\phi)=\int p \mathrm{~d} \phi$.

Proof We first have to check that $\vartheta^{-1}(p)$ is an algebra map $\mathcal{G}(X) \rightarrow[0,1]$. According to Lemma 15 we have to check $\vartheta^{-1}(p)(\phi)=\int\left(\vartheta^{-1}(p) \circ \eta\right) \mathrm{d} \phi$. But this holds by definition, since $\vartheta^{-1}(p)(\eta(x))=\int p \mathrm{~d} \eta(x)=p(x)$.

For naturality assume $f: X \rightarrow \mathcal{G}(Y)$; we get, for an algebra map $g: \mathcal{G}(Y) \rightarrow[0,1]$ and $x \in X$,

$$
\begin{aligned}
\left(\vartheta_{X} \circ\left((-) \circ f^{\$}\right)\right)(g)(x) & =\left(g \circ f^{\$}\right)(\eta(x)) \\
& =g\left(f^{\$}(\eta(x))\right) \\
& =g(f(x)) \\
& =\int \vartheta_{Y}(g) \mathrm{d} f(x) \quad \text { by Lemma } 15 \\
& {[10] } \\
& =f^{*}\left(\vartheta_{Y}(g)\right)(x) \\
& =\left(f^{*} \circ \vartheta_{Y}\right)(g)(x) .
\end{aligned}
$$

Finally, $\vartheta$ and $\vartheta^{-1}$ really are each others inverses:

$$
\begin{aligned}
\left(\vartheta \circ \vartheta^{-1}\right)(p)(x) & =\vartheta^{-1}(p)(\eta(x)) \\
& =\int p \mathrm{~d} \eta(x) \\
& =p(x) \\
\left(\vartheta^{-1} \circ \vartheta\right)(g)(\phi) & =\int \vartheta(g) \mathrm{d} \phi \\
& =g(\phi) \text { by Lemma } 15
\end{aligned}
$$

The discrete analogue of Lemma 16 says $\operatorname{Conv}(\mathcal{D}(X),[0,1]) \cong[0,1]^{X}$, where $\operatorname{Conv}=\mathcal{E} \mathcal{M}(\mathcal{D})$ is the category of convex sets. This result holds because $\mathcal{D}(X)$ is the free convex set on $X \in$ Sets. The quantum analogue is $\operatorname{Conv}(\mathcal{D M}(H),[0,1]) \cong \mathcal{E} f(H)$, see [16], [17].

\section{PREDicates AND StATES}

We first show that by "homming into $[0,1]$ " we can get from effect modules to Eilenberg-Moore algebras.

Lemma 17: For an effect module $E \in$ EMod one can turn the homset $\operatorname{EMod}(E,[0,1])$ into a measurable space, by providing it with the least $\sigma$-algebra making all evaluation maps $\mathrm{ev}_{x}: \operatorname{EMod}(E,[0,1]) \rightarrow[0,1]$ measurable, where $\mathrm{ev}_{x}(h)=h(x)$ for $x \in E$.

This homset $\operatorname{EMod}(E,[0,1])$ then carries an EilenbergMoore algebra structure:

$$
\begin{aligned}
& \mathcal{G}(\operatorname{EMod}(E,[0,1])) \stackrel{\alpha_{E}}{\longrightarrow} \operatorname{EMod}(E,[0,1]) \\
& \psi \longmapsto \lambda x \in E . \int \mathrm{ev}_{x} \mathrm{~d} \psi \text {. }
\end{aligned}
$$

Each map $f: E \rightarrow D$ of effect modules yields an algebra $\operatorname{map}(-) \circ f: \operatorname{EMod}(D,[0,1]) \rightarrow \operatorname{EMod}(E,[0,1])$. Thus we obtain a functor $\operatorname{EMod}(-,[0,1]): \operatorname{EMod}^{\text {op }} \rightarrow \mathcal{E M}(\mathcal{G})$.

Proof We check the algebra equations:

$$
(\alpha \circ \eta)(h)(x)=\int \mathrm{ev}_{x} \mathrm{~d} \eta(h)=\mathrm{ev}_{x}(h)=h(x)
$$




$$
\begin{aligned}
(\alpha \circ \mu)(\Psi)(x) & =\int e v_{x} \mathrm{~d} \mu(\Psi) \\
& =\int\left(\lambda \psi \cdot \int e v_{x} \mathrm{~d} \psi\right) \mathrm{d} \Psi \quad \text { by Lemma } 8 \\
& =\int \alpha(-)(x) \mathrm{d} \Psi \\
& =\int\left(e v_{x} \circ \alpha\right) \mathrm{d} \Psi \\
& \underline{\underline{6}} \int \mathrm{e} v_{x} \mathrm{~d} \mathcal{G}(\alpha)(\Psi) \\
& =\alpha(\mathcal{G}(\alpha)(\Psi))(x) \\
& =(\alpha \circ \mathcal{G}(\alpha))(\Psi)(x) .
\end{aligned}
$$

As to functoriality, for $\Psi \in \mathcal{G}(\operatorname{EMod}(D,[0,1]))$ and $x \in E$,

$$
\begin{aligned}
& \left(\alpha_{E} \circ \mathcal{G}((-) \circ f)\right)(\Psi)(x) \\
& =\alpha_{E}(\mathcal{G}((-) \circ f)(\Psi))(x) \\
& =\int \mathrm{e} v_{x} \mathrm{~d} \mathcal{G}((-) \circ f)(\Psi) \\
& =\int \mathrm{e} v_{x} \circ((-) \circ f) \mathrm{d} \Psi \\
& =\int\left(\lambda h \in \mathbf{E M o d}(D,[0,1]) \cdot \mathrm{e} v_{x}(h \circ f)\right) \mathrm{d} \Psi \\
& =\int(\lambda h \cdot h(f(x))) \mathrm{d} \Psi \\
& =\int e v_{f(x)} \mathrm{d} \Psi \\
& =\alpha_{D}(\Psi)(f(x)) \\
& =\left(\alpha_{D}(\Psi) \circ f\right)(x) \\
& =\left(((-) \circ f) \circ \alpha_{D}\right)(\Psi)(x) .
\end{aligned}
$$

We turn to Eilenberg-Moore algebras. Let $\beta: \mathcal{G}(Y) \rightarrow Y$ be an arbitrary algebra, for $Y \in$ Meas. We do not need to expand on the structure induced on $Y$ by this map $\beta$; instead we only briefly mention what $\beta$ does (see also [13]). For each probability measure $\phi \in \mathcal{G}(Y)$, the value $\beta(\phi) \in Y$ is the barycenter of $\phi:$ it satisfies for each predicate $q: Y \rightarrow[0,1]$ that is an algebra map:

$$
q(\beta(\phi))=\int q \mathrm{~d} \phi
$$

Indeed, since $q$ is a homomorphism of algebras:

$$
\begin{aligned}
q(\beta(\phi))= & \alpha(\mathcal{G}(q)(\phi)) \\
& \text { where } \alpha: \mathcal{G}([0,1]) \rightarrow[0,1] \text { is as in Lemma } 15 \\
= & \int \operatorname{id~} \mathrm{d} \mathcal{G}(q)(\phi) \stackrel{6}{=} \int q \mathrm{~d} \phi .
\end{aligned}
$$

In fact, a predicate $q: Y \rightarrow[0,1]$ is an algebra map if and only if (15) holds (for each $\phi$ ).

Lemma 18: Let $\beta: \mathcal{G}(Y) \rightarrow Y$ be an Eilenberg-Moore algebra. We write $\operatorname{HPred}(Y)$ for the homset of algebra homomorphisms $q: Y \rightarrow[0,1]$. These maps form an effect module.

For each algebra map $g: Y \rightarrow Z$ pre-composition $(-) \circ g$ with $g$ yields a map of effect modules $\operatorname{HPred}(Z) \rightarrow \operatorname{HPred}(Y)$.

Thus we obtain a functor HPred: $\mathcal{E M}(\mathcal{G}) \rightarrow$ EMod $^{\text {op }}$.

Proof We rely on the characterisation (15).

- The zero and one maps $\mathbf{1}_{\emptyset}, \mathbf{1}_{Y} \in \operatorname{Pred}(Y)$ satisfy (15, and are thus in $\operatorname{HPred}(Y)$.

$$
\begin{aligned}
1_{\emptyset}(\beta(\phi)) & =0=\phi(0)=\int \mathbf{1}_{\emptyset} \mathrm{d} \phi \\
1_{Y}(\beta(\phi)) & =1=\phi(Y)=\int \mathbf{1}_{Y} \mathrm{~d} \phi .
\end{aligned}
$$

- If $p \in \operatorname{HPred}(Y)$, then also $p^{\perp}=\mathbf{1}_{Y}-p=\lambda y$. $1-p(y)$ since:

$$
\begin{aligned}
p^{\perp}(\beta(\phi)) & =1-p(\beta(\phi)) \\
& =\left(\int \mathbf{1}_{Y} \mathrm{~d} \phi\right)-\left(\int p \mathrm{~d} \phi\right) \\
& =\int\left(\mathbf{1}_{Y}-p\right) \mathrm{d} \phi=\int p^{\perp} \mathrm{d} \phi .
\end{aligned}
$$

Remaining cases for $\oslash, s \bullet(-)$ and $\bigvee_{n}$ are left to the reader.

We turn to algebra maps. Let $g:(\mathcal{G}(Y) \stackrel{\beta}{\rightarrow} Y) \longrightarrow$ $(\mathcal{G}(Z) \stackrel{\gamma}{\rightarrow} Z)$ be a homomorphism of algebras, commuting as in $\gamma \circ \mathcal{G}(g)=g \circ \beta$. Then $p \circ g$ is in $\operatorname{HPred}(Y)$ if $p \in \operatorname{HPred}(Z)$, since for $\phi \in \mathcal{G}(Y)$,

$$
\begin{aligned}
(p \circ g)(\beta(\phi)) & =(p \circ g \circ \beta)(\phi) \\
& =(p \circ \gamma \circ \mathcal{G}(g))(\phi) \\
& =p(\gamma(\mathcal{G}(g)(\phi))) \\
& =\int p \mathrm{~d} \mathcal{G}(g)(\phi) \quad \text { because } p \in \operatorname{HPred}(Z) \\
& \underline{\underline{6}} \int(p \circ g) \mathrm{d} \phi .
\end{aligned}
$$

Theorem 19: The two functors from Lemmas 17 and 18 form an adjunction $H$ Pred $\dashv \operatorname{EMod}(-,[0,1])$ in:

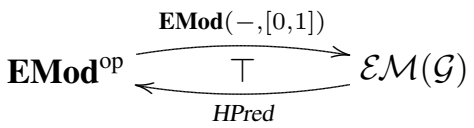

Proof For $E \in \operatorname{EMod}$ and $(Y, \beta) \in \mathcal{E M}(\mathcal{G})$ we have to establish a bijective correspondence between:

$$
\begin{array}{ll}
\frac{Y_{\stackrel{f}{\longrightarrow} \operatorname{EMod}(E,[0,1])}}{E \underset{g}{\longrightarrow} \operatorname{HPred}(Y)} & \text { in } \mathcal{E M}(\mathcal{G}) \\
\text { in } \mathbf{E M o d}
\end{array}
$$

The correspondence is given in the standard way by variableswapping. We need to check that the relevant conditions hold.

- Given $f$ as above, take $\bar{f}(x)(y)=f(y)(x)$. We first check that $\bar{f}(x) \in \operatorname{HPred}(Y)$, via condition (15):

$$
\begin{array}{rlr}
\bar{f}(x)(\beta(\phi)) & =f(\beta(\phi))(x) & \\
& =\alpha_{E}(\mathcal{G}(f)(\phi))(x) & f \text { is an algebra map } \\
& =\int e v_{x} \mathrm{~d} \mathcal{G}(f)(\phi) & \text { see Lemma 17 } \\
& \text { ㅌ } \int\left(\mathrm{ev}_{x} \circ f\right) \mathrm{d} \phi & \\
& =\int \bar{f}(x) \mathrm{d} \phi .
\end{array}
$$

It is easy to see that $\bar{f}$ is a map of effect algebras.

- In the reverse direction we have $\bar{g}(y)(x)=g(x)(y)$. This $\bar{g}(y)$ is clearly a map of effect algebras. And $\bar{g}$ is an 
algebra map, since:

$$
\begin{aligned}
& \left(\alpha_{E} \circ \mathcal{G}(\bar{g})\right)(\phi)(x) \\
& =\alpha_{E}(\mathcal{G}(\bar{g})(\phi))(x) \\
& =\int \mathrm{ev}_{x} \mathrm{~d} \mathcal{G}(\bar{g})(\phi) \quad \text { see Lemma 17 } \\
& \underline{\underline{6}} \int\left(\mathrm{ev}_{x} \circ \bar{g}\right) \mathrm{d} \phi \\
& =\int g(x) \mathrm{d} \phi \\
& =g(x)(\beta(\phi)) \quad \text { since } g(x) \in \operatorname{HPred}(Y) \\
& =\bar{g}(\beta(\phi))(x) \\
& =(\bar{g} \circ \beta)(\phi)(x) .
\end{aligned}
$$

By combining the previous result with Lemmas 14 and 16 we establish the same situation described in [3] for classical, discrete probabilistic logic, and quantum logic, but now for continuous probabilistic logic. It is in fact Theorem 1 from the Introduction.

Corollary 20: The two triangles below commute, up-toisomorphism,

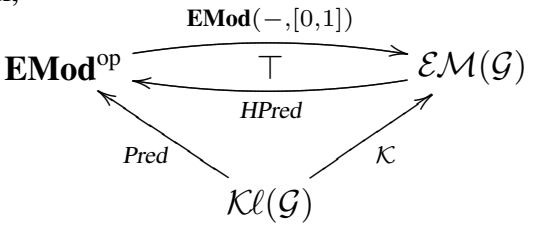

where $\mathcal{K}$ is the standard (full and faithful) "comparison" functor inserting the Kleisli category of a monad in its category of algebras.

In the Introduction we started with Dijkstra's weakest precondition calculus, in terms of bijective correspondences (1). These same correspondences, for computations $X \rightarrow Y$ on measurable spaces, are a consequence of the previous result:

\begin{tabular}{cl}
$\frac{X \longrightarrow \mathcal{G}(Y)}{\mathcal{G}(X) \longrightarrow \mathcal{G}(Y)}$ & i.e as Kleisli maps \\
\hline \hline $\operatorname{Pred}(Y) \longrightarrow \operatorname{Pred}(X)$ & i.e as algebra maps
\end{tabular}

The last map gives the weakest precondition operation $\mathrm{wp}(f): \operatorname{Pred}(Y) \rightarrow \operatorname{Pred}(X)$ corresponding to $f: X \rightarrow$ $\mathcal{G}(Y)$. It is given by substitution $f^{*}$ from Definition 5. In the reverse direction, starting from $W: \operatorname{Pred}(Y) \rightarrow \operatorname{Pred}(X)$ in EMod we get a computation $c(W): X \rightarrow \mathcal{G}(Y)$ by:

$$
\begin{aligned}
c(W)(x) & =\lambda N \in \Sigma_{Y} \cdot W\left(\mathbf{1}_{N}\right)(x) \\
& =\theta_{Y}(W(-)(x)), \quad \text { see Lemma 14. }
\end{aligned}
$$

This correspondence is essentially a reformulation of the duality of Kozen [4]: we restrict ourselves to maps going into $[0,1]$, where Kozen uses bounded maps.

\section{FINAL REMARKS}

Somewhat remarkably, the proof of the adjunction $\operatorname{EMod}^{\text {op }} \leftrightarrows \mathcal{E} \mathcal{M}(\mathcal{G})$ in Theorem 19 does not require a precise characterisation of the category of algebras $\mathcal{E M}(\mathcal{G})$ of the Giry monad. One such characterisation is elaborated in [24]. A closer connection to the category of convex compact Hausdorff spaces, used in [23], see als [25], will be elaborated in an extended version of the current paper.

\section{REFERENCES}

[1] E. Dijkstra, "Guarded commands, nondeterminacy and formal derivation of programs," Communications of the ACM, vol. 18(8), pp. 453-457, 1975.

[2] T. Heinosaari and M. Ziman, The Mathematical Language of Quantum Theory. From Uncertainty to Entanglement. Cambridge Univ. Press, 2012.

[3] B. Jacobs, "New directions in categorical logic, for classical, probabilistic and quantum logic," 2012, see arxiv.org/abs/1205.3940.

[4] D. Kozen, "Semantics of probabilistic programs," Journ. Comp. Syst. Sci, vol. 22(3), pp. 328-350, 1981.

[5] F. Lawvere, "Metric spaces, generalized logic, and closed categories," Seminario Matematico e Fisico. Rendiconti di Milano, vol. 43, pp. 135166, 1973.

[6] M. Giry, "A categorical approach to probability theory," in Categorical Aspects of Topology and Analysis, ser. Lect. Notes Math., B. Banaschewski, Ed., no. 915. Springer, Berlin, 1982, pp. 68-85.

[7] C. Jones and G. Plotkin, "A probabilistic powerdomain of evaluations," in Logic in Computer Science, IEEE. Computer Science Press, 1989, pp. 186-195.

[8] J. Desharnais, A. Edalat, and P. Panangaden, "Bisimulation for labeled Markov processes," Inf. \& Comp., vol. 179(2), pp. 163-193, 2002.

[9] R. Tix, K. Keimel, and G. Plotkin, Semantic Domains for Combining Probability and Non-Determinism, ser. Elect. Notes in Theor. Comp. Sci. Elsevier, Amsterdam, 2005, no. 129.

[10] V. Danos, J. Desharnais, F. Laviolette, and P. Panangaden, "Bisimulation and cocongruence for probabilistic systems," Inf. \& Comp., vol. 204, pp. 503-523, 2006.

[11] D. Varacca and G. Winskel, "Distributing probability over nondeterminism,” Math. Struct. in Comp. Sci., vol. 16, pp. 87-113, 2006.

[12] K. Keimel, "The monad of probability measures over compact ordered spaces and its Eilenberg-Moore algebras," Topology and its Applications, vol. 156, pp. 227-239, 2008.

[13] — - "Abstract ordered compact convex sets and algebras of the (sub)probabilistic power domain monad over ordered compact spaces," Algebra an Logic, vol. 48(5), pp. 330-343, 2009.

[14] P. Panangaden, Labelled Markov Processes. Imperial College Press, 2009.

[15] K. Keimel, A. Rosenbusch, and T. Streicher, "Relating direct and predicate transformer partial correctness semantics for an imperative probabilistic-nondeterministic language," Theor. Comp. Sci., vol. 412 , pp. 2701-2713, 2011.

[16] P. Busch, "Quantum states and generalized observables: a simple proof of Gleason's Theorem,” Phys. Review Letters, vol. 91(12):120403, pp. $1-4,2003$.

[17] B. Jacobs and J. Mandemaker, "Relating operator spaces via adjunctions," in Logic and Algebraic Structures in Quantum Computing and Information, ser. Lect. Notes in Logic, J. C. Reimann, V. Harizanov, and A. Eskandarian, Eds. Cambridge Univ. Press, 2013, see arxiv.org/abs/1201.1272.

[18] D. J. Foulis and M. Bennett, "Effect algebras and unsharp quantum logics," Found. Physics, vol. 24(10), pp. 1331-1352, 1994.

[19] A. Dvurečenskij and S. Pulmannová, New Trends in Quantum Structures. Dordrecht: Kluwer Acad. Publ., 2000.

[20] B. Jacobs, Categorical Logic and Type Theory. Amsterdam: North Holland, 1999.

[21] S. Gudder, "Quantum probability," in Handbook of Quantum Logic and Quantum Structures: Quantum Structures, K. Engesser, D. M. Gabbai, and D. Lehmann, Eds., North Holland, Elsevier. Computer Science Press, 2007, pp. 121-146.

[22] H. Reichenbach, The theory of probability. Univ. California Press, 1949.

[23] B. Jacobs and J. Mandemaker, "The expectation monad in quantum foundations," in Quantum Physics and Logic (QPL) 2011, ser. Elect. Proc. in Theor. Comp. Sci., B. Jacobs, P. Selinger, and B. Spitters, Eds., vol. 95, 2012, pp. 143-182.

[24] E.-E. Doberkat, "Eilenberg-Moore algebras for stochastic relations," Inf. \& Comp., vol. 204(12), pp. 1756-1781, 2006, erratum and addendum in: 206(12):1476-1484, 2008.

[25] T. Swirszcz, "Monadic functors and convexity," Bull. de l'Acad. Polonaise des Sciences. Sér. des sciences math., astr. et phys., vol. 22, pp. 39-42, 1974. 